\title{
Mechanical behavior and properties of hydrogen bonded graphene/polymer nano-interfaces
}

\author{
Zhaohe Dai a, b, d , Guorui Wang b, c, Luqi Liu ${ }^{\text {b, ** }}{ }^{\text {, Yuan Hou }}{ }^{\text {b, c }}$, Yueguang Wei ${ }^{\text {a, ***, }}$ \\ Zhong Zhang ${ }^{\mathrm{b}, \mathrm{c}, *}$ \\ a State Key Laboratory of Nonlinear Mechanics, Institute of Mechanics, Chinese Academy of Sciences, Beijing, 100190, China \\ ${ }^{\mathrm{b}}$ CAS Key Laboratory of Nanosystem and Hierachical Fabrication, CAS Center for Excellence in Nanoscience, National Center for Nanoscience and \\ Technology, Beijing, 100190, China \\ c CAS Key Laboratory of Mechanical Behavior and Design of Materials, Department of Modern Mechanics, University of Science and Technology of China, \\ Hefei, 230027, China \\ d University of Chinese Academy of Science, Beijing, 100049, China
}

\section{A R T I C L E I N F O}

\section{Article history:}

Received 16 May 2016

Received in revised form

19 August 2016

Accepted 1 September 2016

Available online 16 September 2016

\section{Keywords:}

Interface

Nano composites

Raman spectroscopy

Modelling

Interfacial strength

\begin{abstract}
A B S T R A C T
There is increasing evidence in literature for significant improvements in both toughness and strength of graphene-based nanocomposites through engineering their nano-interfaces with hydrogen bonds ( $\mathrm{H}-$ bonds). However, the underlying mechanical behaviors and properties of these H-bonded interfaces at the microscopic level were still not experimentally clarified and evaluated. Herein, this work reports a study on the interfacial stress transfer between a monolayer graphene and a commonly used poly(methyl methacrylate) (PMMA) matrix under pristine vdW and modified H-bonding interactions. A nonlinear shear-lag model considering friction beyond linear bonding was proposed to understand evolution of interfacial stresses and further identify key interfacial parameters (such as interfacial stiffness, strength, frictional stress and adhesion energy) with the aid of in situ Raman spectroscopy and atomic force microscopy. The present study can provide fundamental insight into the reinforcing mechanism and unique mechanical behavior of chemically modified graphene nano-interfaces and develop further a basis for interfacial optimal design of graphene-based high-performance nanocomposites.
\end{abstract}

(C) 2016 Published by Elsevier Ltd.

\section{Introduction}

Nanocarbon materials have proven to be remarkable materials because of its unique physical properties [1,2]. In particular, onedimensional carbon nanotubes (CNT) and two dimensional graphene sheets exhibit striking mechanical stiffness (with 1 TPa Young's modulus), ultrahigh aspect ratio $\left(>10^{4}\right)$ and exceptional robustness (failure strain up to $20 \%$ ), making them ideal nanofillers for high-performance nanocomposites [3-5]. From classic view of fiber/matrix system, one of the most critical issues that determine

\footnotetext{
* Corresponding author. CAS Key Laboratory for Nanosystem and Hierachical Fabrication, CAS Center for Excellence in Nanoscience, National Center for Nanoscience and Technology, Beijing, 100190, China.

** Corresponding author.

*** Corresponding author

E-mail addresses: liulq@nanoctr.cn (L. Liu), ywei@lnm.imech.ac.cn (Y. Wei), zhong.zhang@nanoctr.cn (Z. Zhang).
}

the bulk performance, durability and reliability of composites lies in the interfacial properties, which should be substantial to undergo sufficient stress transfer caused by the stiffness mismatch during deformation [6-8]. This issue could be much more critical in nanocarbon-based nanocomposites, where there are orders of magnitude more interfacial area created per volume of filler added and the high stiffness of graphene and carbon nanotube makes such mismatch more distinctive. Thus, in efforts to optimize the bulk performance of nanocarbon-based nanocomposites, particularly central is to figure out the elucidation of specific nanocarbonmatrix adhesions and the mechanical behavior of these nanointerfaces.

Recently, owing to its monolayered nature, graphene with micrometer lateral size has been particularly promising as not only superior nanofiller, but also its own 'wireless' strain sensors with the aid of Raman spectroscopy [9-18]. Through macroscopically applying strain on matrix or substrate, in situ recording of the Raman peaks shift of graphene can provide quantitative 
measurement of strain distributions in the whole graphene sheet and, at the same time, a tool for thorough understanding of nanointerface mechanics as well as their properties [9,11,18]. For instance, Young et al. have monitored the stress transfer in monolayer graphene sandwiched between PMMA and SU-8 polymer layer where interfacial adhesion was taking place though van der Waals (vdW) bonding across an atomically smooth surface. Based on linear shear-lag assumptions used in fiber/matrix interfaces, the derived interfacial shear strength was on the order of 2.3 MPa [9]. Moreover, unlike the fracture behavior of carbon fibers undergoing when matrix was subjected to a larger strain level, stress transfer between the graphene and polymer was still taking place through interfacial friction (in the range 0.3-0.8 $\mathrm{MPa}$ ) [9]. Nanoscale frictional behavior beyond the well-established linear interfacial mechanics in fiber-based composites should thus be carefully considered for pristine graphene-based nano-interfaces, in order to experimentally evaluate their mechanical behavior and properties. Similar results were also revealed through molecular dynamics (MD) simulations about vdW interaction dominated graphene interfaces where adhesion and sliding force would spontaneously take place even without an applied normal pressure [19]. More recently, Zhu et al. also developed a nonlinear shear-lag model by assuming a constant frictional/sliding stress (i.e. shear strength in their analysis) beyond the linear stage and obtained 0.46-0.69 $\mathrm{MPa}$ of interfacial strength or frictional stress for monolayer graphene-polyethylene terephthalate(PET) nanointerfaces [11].

Besides of these elegant insights into the mechanical properties and nonlinear behavior of the graphene-based vdW interfaces, the high aspect ratio of the graphene nanosheet also offers tremendous opportunities for tuning interfacial chemistry at the molecular level, and further enables optimal design of graphene-based nanocomposites by engineering interfacial crosslinks beyond vdW interactions [20]. Especially, previous MD simulations suggested that crosslinking hydrogen bonds $(\mathrm{H}-$ bonds), which were widely utilized in toughening and reinforcing bio-materials (e.g. silk and nacre) [21], in graphene-based interfacial galleries would feature them with much improved mechanical interactions, compared with the pristine weak vdw interactions [22-24]. Recent literature in graphene-based nanocomposites further highlighted such interfacial H-bonds crosslinks by which improvements could be achieved in both bulk toughness and strength [25-30]. However, the underlying mechanical behaviors and properties of these $\mathrm{H}$-bonded interfaces at the microscopic level were still not experimentally clarified and evaluated. Consequently, optimal designs by tuning the $\mathrm{H}-$ bonding within graphene-related bulk materials are limited by missing the key mechanical parameters such as the interfacial strength and frictional stress. Characterization of the mechanical behavior and properties of $\mathrm{H}$-bonded graphene-based interface is thus essential both from a technological perspective for design of high-performance nanocomposite and from a fundamental interest in understanding nano-interface mechanics.

Herein, this work reports a study on the interfacial stress transfer between a monolayer graphene and a commonly poly(methyl methacrylate) (PMMA) matrix under pristine and modified interfacial interactions (i.e. the pristine vdW and H-bonding interaction). A nonlinear shear-lag model considering friction beyond linear bonding and corresponding analytical solutions were presented, offering a simple tool to understand evolution of interfacial (shear) stresses along the graphene/PMMA interface during subsequent deformations. We then further identify key interfacial parameters including interfacial (shear) stiffness, strength and frictional stress for both graphene-based vdW and H-bonding nano-interface with the aid of in situ Raman spectroscopy measurements. Finally, the adhesion energy for these two types of graphene interfaces is calculated on the basis of frictional behavior induced buckles after release. Our nonlinear model might be also valid for graphene nano-interfaces with various types of interfacial interactions (e.g. covalent and coordinative bonds) and interfacial parameters presented in this study can develop valuable basis for interfacial optimal design of high-performance graphene-based nanocomposites.

\section{Experiment and model}

\subsection{Problem definition}

In our experiments, the graphene samples were prepared by micromechanical cleavage and transferred onto the surface of poly(methyl methacrylate) (PMMA) matrix [13]. The monolayer graphene sheet was exposed on top of PMMA matrix, allowing further chemical functionalization. The mechanical deformation was carried out with a PMMA cantilever beam mounted onto a piezoelectric stage with resolution of $600 \mathrm{~nm}$ in Renishaw Raman spectroscope [13,31-37]. Oxidization of individual graphene sheet was performed by using $\mathrm{O}_{3}-\mathrm{H}_{2} \mathrm{O}$ gaseous mixture as oxidizing agent in order to tuning the native interfacial interaction into $\mathrm{H}$ bonding interaction (see Ref. [39] for more details on the experiments) [38,39]. In our analysis, for the sake of symmetry only half of the graphene/matrix system in the horizontal $(x)$ direction is considered as shown in Fig. 1a. The graphene sheet holds a halflength $(L / 2)$ with $x=0$ at the center of the nanoplate. As the applied strain to the matrix experimentally is small $(<1 \%)$, all the materials involved in the problem are assumed to have a linearly elastic behavior and the non-linearity is concentrated at the interface in our modified shear lag model. Thus, the graphene holds a Young's modulus of $E_{g}(=1 \mathrm{TPa})$ [3], the constitutive equation of graphene can be given as:

$\sigma=E_{g} \varepsilon=E_{g} \frac{d u_{g}}{d x}$

Where $\sigma, \varepsilon$ and $u_{g}$ denote the axial stress, strain and displacement in the graphene, respectively. Meanwhile, for the strains of the matrix, the relationship also holds:

$\varepsilon_{m}=\frac{d u_{m}}{d x}$

where $\varepsilon_{m}$ and $u_{m}$ denote the strain and displacement in the matrix. Note that the definitions of $u_{m}$ and $u_{g}$ also yield:

$\delta=u_{m}-u_{g}$

Where $\delta$ is the tangential relative displacement at the graphene nanosheet/matrix interface.

\subsection{Conventional shear-lag assumptions}

Recently, shear lag analysis has been used to describe the shear stress transfer between a monolayer graphene and a substrate or matrix $[9,11,18]$. In this type of linear model, perfect bonding and linear relationship between tangential relative displacement and the interfacial shear stress are assumed for the nano-interfaces. During uniaxial tensile loading, the tensile stress transfers from the substrate to the graphene by means of this interfacial shear stress. Combining Eq. (1), the equilibrium condition for the graphene in the $x$ direction leads to: 
$\tau=-t \frac{d \sigma}{d x}=-E_{g} t \frac{d \varepsilon}{d x}$

where $\tau$ is the interfacial shear stress and $t=0.34 \mathrm{~nm}$ is the thickness of monolayer graphene. In efforts to obtain the final differential equation only about the variable of strain (experimentally measurable via Raman spectroscopy) in Section 3, the first derivative of Eq. (4) is needed here and could yield:

$\frac{d \tau}{d x}=-E_{g} t \frac{d^{2} \varepsilon}{d x^{2}}$

Conventional shear-lag analysis in fiber-reinforced composites assumed linear assumption for interface $(\tau \sim \delta)$, Eq. (5) can hence be easily solved as presented in Section 3.1 by combining Eq. (1) for graphene, Eq. (2) for substrate as well as Eq. (3) for interfaces $[9,11,18]$. As only shear stress arises in our graphene/matrix system (Fig. 1a), for the sake of simplicity, the interfacial shear stress (traction), $\tau$, will be equivalently indicated as "interfacial stress"; the interfacial tangential relative displacement (separation) across the interface, $\delta$, will be equivalently termed "relative displacement".

\subsection{Nonlinear shear-lag model}

In efforts to tackle analytical modelling of the nano-interfaces that featured unique frictional behavior, the linear interfacial behavior ( $\tau-\delta$ relationship) in conventional shear-lag analysis was modified after considering frictional forces beyond elastic bonding. This interface could be thought of as a thin layer consisting of uniformly distributed interfacial bonding and having a nonlinear constitutive behavior under shear deformation. As shown in Fig. 1b, during initially elastic bonding stage, the interfacial stress in this thin layer increases linearly with a constant stiffness $\left(k_{e}\right)$ in response to the interfacial relative displacement (shear-lag analysis), until reaching the so-called critical interfacial strength $\left(\tau_{c}\right)$. Beyond linear stage, there is a frictional stage with considerable sliding stress compared to the interfacial strength. Here we assume that the interfacial frictional
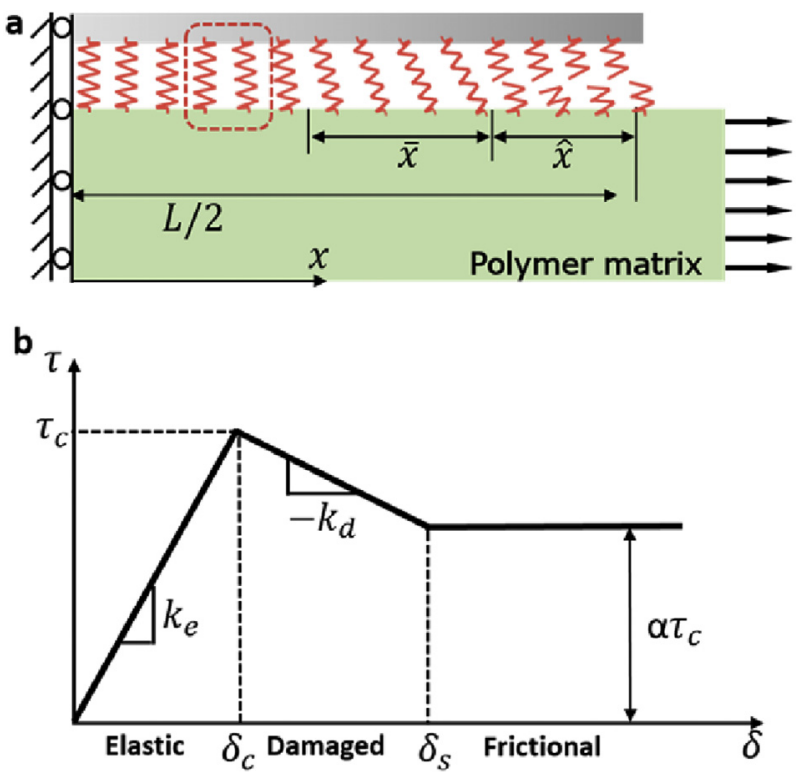

Fig. 1. (a) Schematic diagram of the monolayer graphene sheet/polymer configuration under uniaxial tension. (b) Nonlinear shear-lag model. stress keeps a constant of $\alpha \tau_{c}$, and $\alpha(\leq 1)$ represents the ratio of frictional stress to interfacial strength. In addition, when the friction stress could not remain the level of interfacial strength $(\alpha<1)$, an intermediate stage, behaves like interfacial "softening", is essential for the continuity of interfacial stresses between the linear and frictional stage. We define this intermediate stage as damaging stage, describing the transition stage of interfacial bond breaking (when interfacial stress reaches bonding strength) to steady interfacial sliding (bond breaking and re-forming caused frictional behavior), where interfacial stress decreases with another constant slope $\left(-k_{d}\right)$. The following analytical relationships represent the three branches of nonlinear $\tau-\delta$ relationship:

$\tau=\left\{\begin{array}{l}k_{e} \delta ; \quad \text { for } 0 \leq \delta \leq \frac{\tau_{c}}{k_{e}} \\ \tau_{c}+k_{d}\left(\frac{\tau_{c}}{k_{e}}-\delta\right) ; \text { for } \frac{\tau_{c}}{k_{e}} \leq \delta \leq \frac{\tau_{c}}{k_{e}}+(1-\alpha) \frac{\tau_{c}}{k_{d}} \\ \alpha \tau_{c} ; \quad \text { for } \delta \geq \frac{\tau_{c}}{k_{e}}+(1-\alpha) \frac{\tau_{c}}{k_{d}}\end{array}\right.$

where the three branches of the relationship can respectively be labeled as elastic, damaging, and frictional.

It is worth noting that our nonlinear model proposed here could be easily simplified to previous interfacial mechanics models including: i) when $k_{e}=\infty$ and $\alpha=1$, constant interfacial stress analysis or tension-shear chain model (describing interfacial sliding in pull-out of carbon nanotube or "sacrificial bonds" in biological materials) [40,41]; ii) when $k_{d}=\infty$ and $\alpha=0$, linear shear-lag (describing stress transfer in carbon fiber) [42]; iii) when $\alpha=0$, bilinear cohesive zone models (describing the crack initiation and propagation in fiber-polymer, coating adhesives and bimaterial interfaces) [43]; iv) when $\alpha=1$, nonlinear shear-lag models (describing interfacial sliding behavior in graphenebased vdW interfaces) [11]. In fact, there is inconsistence in interfacial frictional stress to strength ratio in previous experimental work about vdW interface $[9,11]$. In addition, different types of interfacial crosslinks also showed probably different frictional behavior $[44,45]$. For instance, though no experimental results exist, according to MD simulations results, the covalent bonded graphene interfaces were examined to exhibit a smaller $\alpha$ (interfacial frictional stress to strength ratio) compared with ionic, hydrogen bonded or vdW interfaces [45]. Our model thus takes account of diverse relationships between the interfacial shear stress and interfacial relative displacement of various types of crosslinking interfaces, by which complex nanocarbon-matrix adhesions will be experimentally clarified and evaluated in future. Here, to focus on evolution of interfacial (shear) stresses along the graphene/PMMA interface during subsequent deformations, we set $k_{d}=k_{e}=$ finite, $\alpha=0.5$ in the following discussions without loss of generality in Fig. 2. And then these interfacial parameters of $\mathrm{H}$-bonded and vdW interfaces are identified and compared based experimental results as discussed in Section 4.2.

\section{Analysis of the interfacial shear stresses}

\subsection{Elastic (E) stage}

At small applied strain $\left(\varepsilon_{m}\right)$ on matrix, the whole length of the interface is in elastic conditions, and thus the interfacial behavior is described by the first branch of the nonlinear interfacial stressrelative displacement relationship. Eq. (6)a by using Eq. (3) can be expressed as: 
$\tau_{e}=k_{e}\left(u_{m}-u_{g}\right)$

where the subscript " $e$ " is introduced to refer to the interfacial stress under elastic condition. Deriving Eq. (6) and combing the resulting expression with Eqs. (1)-(2), the following equation is easily found:

$\frac{d \tau_{e}}{d x}=k_{e}\left(\varepsilon_{m}-\varepsilon\right)$

When Eq. (5) is substituted into Eq. (8), the following differential equation is obtained:

$\frac{d^{2} \varepsilon}{d x^{2}}-\beta^{2} \varepsilon+\beta^{2} \varepsilon_{m}=0$

Where $\beta=\sqrt{k_{e} / E_{g} t}$ is the parameter in conventional shear lag analysis [42]. Applying the boundary conditions $\varepsilon=0$ at $x=L / 2$ and $d \varepsilon / d x=0$ at $x=0$ (stemming from symmetry), Eq. (9) can be solved as:

$\varepsilon=\varepsilon_{m}\left(1-\frac{\cosh (\beta x)}{\cosh (\beta L / 2)}\right)$

Substituting Eq. (10) into Eq. (4), the consequent expression of the interfacial shear stress is:

$\tau_{e}=\beta E_{g} t \varepsilon_{m} \frac{\sinh (\beta x)}{\cosh (\beta L / 2)}$

and it is shown in Fig. 2a. Eq. (11) was the same as the shear lag analysis which has been widely used in interfacial stress analysis of fiber-reinforced composites. The maximum interfacial stress gived by Eq. (11) is reached at the end of the graphene sheets (at $x=L / 2$ ). As the applied strain to matrix increase, the interfacial relative displacement increases. The interface is at the elastic stage until $\tau_{e}(x=L / 2)$ reaches a critical interfacial strength, $\tau_{c}$, i.e. the strength of interfacial crosslinking bonds and the applied strain reaches the value:

$\varepsilon_{c}=\frac{\tau_{c}}{\beta E_{g} t \tanh (\beta L / 2)}$

For $\varepsilon_{m}=\varepsilon_{c}$, at the end of the graphene sheet it is $\tau_{e}(x=L / 2)=\tau_{c}$, as shown in Fig. 2b, and damaging (breaking of bonds) starts.

\subsection{Elastic-damaging (E-S) stage}

As the applied strain to matrix further processes after reaching $\varepsilon_{c}$, the graphene/matrix interface enters the elastic-damaging stage: an increasing length $(\bar{x})$ near the edge becomes the damaging condition, while the rest remains in elastic condition as shown in Fig. 2c. For the elastic zone $(0 \leq x \leq L / 2-\bar{x})$, the governing differential equation (9) continue to hold. At the point of $x=L / 2-\bar{x}$, the interfacial stress $\tau_{e}(L / 2-\bar{x})$ is exactly equal to $\tau_{c}$. After combining this boundary condition with Eqs. (4) and (12), with another condition $d \varepsilon / d x=0$ at $x=0$ (stemming from symmetry), the axial strain of graphene in the elastically bonded phase could be solved:

$\varepsilon=\varepsilon_{m}-\frac{\varepsilon_{c} \tanh (\beta L / 2) \cosh (\beta x)}{\sinh [\beta(L / 2-\bar{x})]}$

Substituting Eqs. (13) and (12) into Eq. (4), the consequent expression of the interfacial stress is:
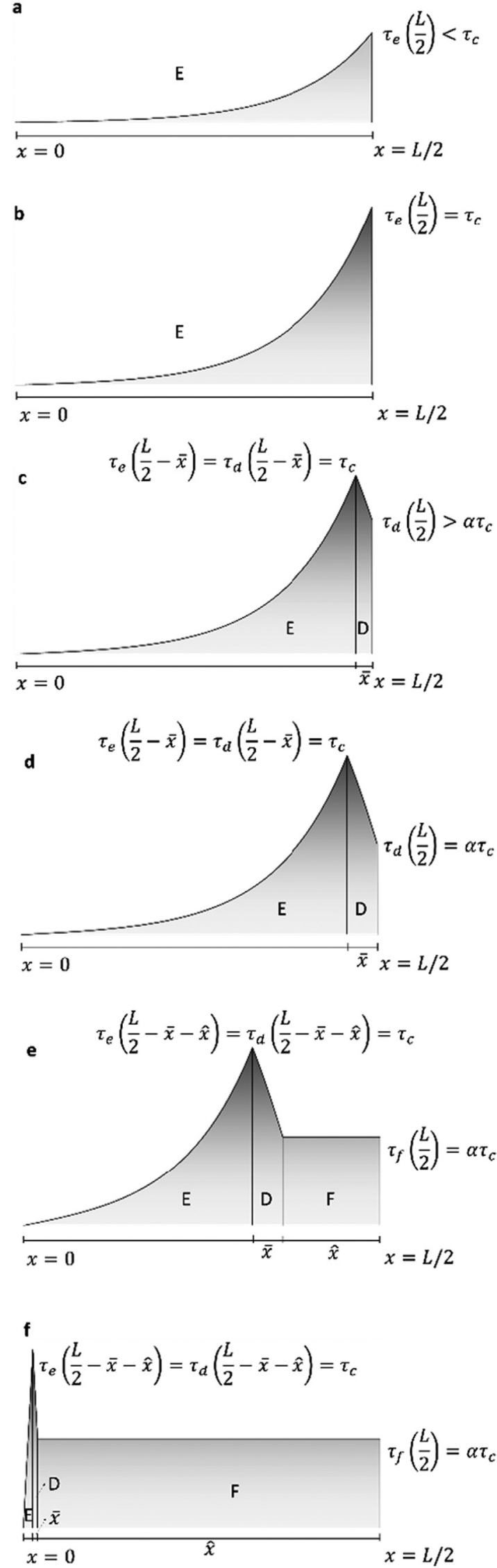

Fig. 2. Interfacial shear stress distribution: (a) E stage; (b) end of E stage; (c) E-D stage; (d) end of E-D stage; (e) E-D-F stage and (f) advanced E-D-F stage. 
$\tau_{e}=\frac{\tau_{c} \sinh (\beta x)}{\sinh [\beta(L / 2-\bar{x})]}$

For the damaging zone $(L / 2-\bar{x} \leq x \leq L / 2)$, the interfacial behavior is described by the second branch of the nonlinear model. In this case, deriving Eq. (6)b and substituting it into Eq. (5) with the aid of Eqs. (1)-(3), the following differential equation governing the damaging zone could be easily found:

$\frac{d^{2} \varepsilon}{d x^{2}}+\gamma^{2} \varepsilon-\gamma^{2} \varepsilon_{m}=0$

where $\gamma=\sqrt{k_{d} / E_{g} t}$. Applying the boundary condition $\varepsilon=0$ at $x=L / 2$ and $\frac{d \varepsilon^{+}(L / 2-\bar{x})}{d x}=\frac{d \varepsilon^{-}(L / 2-\bar{x})}{d x}$ (stemming from continuity of interfacial stress), this differential equation could be solved:

$\varepsilon=\varepsilon_{m}+\frac{\beta}{\gamma} \varepsilon_{C} \tanh (\beta L / 2)[A \cos (\gamma x)+B \sin (\gamma x)]$

where

$$
A=-\frac{\gamma E_{g} t \varepsilon_{m} \cos [\gamma(L / 2-\bar{x})]}{\tau_{c} \cos (\gamma \bar{x})}+\frac{\sin (\gamma L / 2)}{\cos (\gamma \bar{x})}
$$

$B=-\frac{\gamma E_{g} t \varepsilon_{m} \sin [\gamma(L / 2-\bar{x})]}{\tau_{c} \cos (\gamma \bar{x})}-\frac{\cos (\gamma L / 2)}{\cos (\gamma \bar{x})}$. Substituting Eqs. (16) and (12) into Eq. (4), the consequent expression of the interfacial stress is:

$\tau_{d}=\tau_{c}[A \sin (\gamma x)-B \cos (\gamma x)]$

where the subscript " $d$ " is introduced to refer to the interfacial stress under damaging condition. Moreover, by applying the continuity of axial strain of graphene $\varepsilon^{+}(L / 2-\bar{x})=\varepsilon^{-}(L / 2-\bar{x})$ by Eqs. (13) and (16), the following implicit expression is obtained for the length of the damaging zone $\bar{x}$ :

$\frac{\varepsilon_{m}}{\varepsilon_{C}}=\frac{\beta}{\gamma} \tanh (\beta L / 2) \sin (\gamma \bar{x})+\frac{\cos (\gamma \bar{x})}{\tanh [\beta(L / 2-\bar{x})]} \tanh (\beta L / 2)$

Eq. (18) provides the relationship between the applied strain and damaging length at the elastic-damaging stage. As depicted in Fig. $2 \mathrm{~d}$, this stage ends when $\tau_{d}(L / 2)=\alpha \tau_{c}$ and frictional behavior starts. The applied strain needed for the onset of elastic-damagingfrictional stage could be given from Eq. (17):

$\frac{\varepsilon_{m}}{\varepsilon_{c}}=\frac{\beta \tanh (\beta L / 2)}{\gamma \sin (\gamma \bar{x})}-\frac{\alpha \beta \tanh (\beta L / 2)}{\gamma \tan (\gamma \bar{x})}$

Combining Eqs. (18) and (19) can compute the value of damaging length $\bar{x}$ at the end of elastic-damaging stage.

\subsection{Elastic-damaging-frictional ( $E-D-F)$ stage}

As the applied strain to matrix further processes, the graphene/ matrix interface enters the elastic-damaging-frictional stage: an increasing length $(\widehat{x})$ near the edge becomes the frictional condition, while the rest remains in partially damaging and partially elastic condition as shown in Fig. 2 e.

For the elastic zone $(0 \leq x \leq L / 2-\bar{x}-\widehat{x})$, the length of damaging part of interface could be considered as $(\bar{x}+\widehat{x})$ without disturbing boundary condition for the solution of governing differential equation (9). Thus the axial strain of graphene in the elastically bonded phase could be solved by substituting $\bar{x}$ with $(\bar{x}+\widehat{x})$ in Eq. (13):

$\varepsilon=\varepsilon_{m}-\frac{\varepsilon_{c} \tanh (\beta L / 2) \cosh (\beta x)}{\sinh [\beta(L / 2-\bar{x}-\widehat{x})]}$

the consequent expression of the interfacial stress is: $\tau_{e}=\frac{\tau_{c} \sinh (\beta x)}{\sinh [\beta(L / 2-\bar{x}-\widehat{x})]}$

For the damaging zone $(L / 2-\bar{x}-\widehat{x} \leq x \leq L / 2-\widehat{x})$, the governing differential equation (15) continue to hold. At the point of $x=L / 2-\bar{x}$ and $x=L / 2-\bar{x}-\widehat{x}$, the interfacial stress is exactly equal to $\tau_{c}$ and $\alpha \tau_{c}$, respectively. After combining this two boundary condition with Eqs. (4) and (12), the axial strain of graphene in the damaging phase could be solved:

$\varepsilon=\varepsilon_{m}+\frac{\beta}{\gamma} \varepsilon_{C} \tanh (\beta L / 2)[C \cos (\gamma x)+D \sin (\gamma x)]$

where

$$
C=\frac{\alpha \cos [\gamma(L / 2-\bar{x}-\widehat{x})]}{\sin (\gamma \bar{x})}-\frac{\cos [\gamma(L / 2-\bar{x})]}{\sin (\gamma \bar{x})}
$$

and $D=\frac{\alpha \cos [\gamma(L / 2-\bar{x}-\widehat{x})]}{\sin (\gamma \bar{x})}-\frac{\cos [\gamma(L / 2-\bar{x})]}{\sin (\gamma \bar{x})}$. Substituting Eqs. (22) and (12) into Eq. (4), the consequent expression of the interfacial stress is:

$\tau_{d}=\tau_{c}[C \sin (\gamma x)-D \cos (\gamma x)]$

For the frictional zone $(L / 2-\widehat{x} \leq x \leq L / 2)$, the interfacial behavior is described by the third branch of the nonlinear model. Unlike the debonding assumption for micron-size fiber/polymer interface in conventional interfacial model, the graphene/polymer interface could still keep its capacity of transferring loads due to frictional forces (stemming from the breaking and re-forming process of cross-linked bonds or vdW interaction). The sliding stress $\left(\alpha \tau_{c}\right)$ is assumed to be $\alpha$-fold of the interfacial strength in our nonlinear model:

$\tau_{f}=\alpha \tau_{c}$

where the subscript " $f$ " is introduced to refer to the interfacial stress under frictional condition. Applying the boundary condition $\varepsilon=0$ at $x=L / 2$ and combining Eq. (12), the axial strain of graphene in the frictional phase could be obtained by integrating Eq. (5) as:

$\varepsilon=\alpha \varepsilon_{C} \tanh (\beta L / 2)(L / 2-x)$

Moreover, by applying the continuity of axial strain of graphene $\varepsilon^{+}(L / 2-\bar{x}-\widehat{x})=\varepsilon^{-}(L / 2-\bar{x}-\widehat{x})$ by Eqs. (20) and (22) as well as $\varepsilon^{+}(L / 2-\widehat{x})=\varepsilon^{-}(L / 2-\widehat{x})$ by Eqs. (22) and (25), the following implicit expression is obtained for the length of the damaging zone $\bar{x}$ and frictional zone $\hat{x}$ :

$\alpha-\cos (\gamma \bar{x})+\frac{\gamma}{\beta} \sin (\gamma \bar{x}) \tanh [\beta(L / 2-\bar{x}-\widehat{x})]=0$

$\frac{\gamma \varepsilon_{m}}{\beta \varepsilon_{C} \tanh (\beta L / 2)}=\alpha \gamma \widehat{x}+\frac{[1-\alpha \cos (\gamma \bar{x})]}{\sin (\gamma \bar{x})}$

\section{Results and discussion}

\subsection{Evolution of interfacial stresses and strain distribution}

With the interface identified in Sec. 3.1-3, the progressive configuration of the interfacial stresses as well as axial strain of monolayer graphene could be easily derived, offering a simple tool to understand evolution of interfacial stresses and further identify key interfacial parameters based experimental results. The main steps are outlined here. (i) During the elastic stage, the interfacial stresses are given by Eq. (11) along the whole length of the graphene/polymer bonded interface as shown in Fig. 2a. The strain of graphene could be obtained by Eq. (10). Both the stress and strain 
are proportional to the applied strain to the matrix due to the linearly elastic transfer of interface. Note that the maximum interfacial stress occurs at the edges. This stage ends when the applied strain satisfies Eq. (12), where bond breaking occurs at the edge of interface (the interfacial stress reaches to interfacial strength in Fig. 2b). (ii) During the elastic-damaging stage, the behavior of the interface can be followed by gradually increasing length of interfacial bonds breaking zone. For each value of the damaging length, the corresponding applied strain to the matrix can be given by Eq. (18). Then, the interfacial stress distribution is computed by Eqs. (14) and (17) as shown in Fig. 2c. Also, the strain of graphene could be obtained by Eqs. (13) and (16). Note that the interfacial stress still maximizes at end of elastic part and the starts softening to the edge in damaging part. This stage ends when the interfacial stress at the edge decreased to the frictional stress (Fig. 2d). And the applied strain and length of damaging region could be given by the combination of Eqs. (18) and (19). (iii) During the elastic-damagingfrictional stage, the controlling behavior becomes the propagation of the sliding region from the edge to the center of interface. For each value of applied strain, the corresponding damaging and frictional region can be given by combining Eqs. (26) and (27). Then, the interfacial stress distribution is computed by Eqs. (21), (23) and (24) as shown in Fig 2e. Also, the strain of graphene could be obtained by Eqs. (20), (22) and (25). In fact, the E-D-F stage progresses with a gradual increase in the length of the sliding region and a decrease in both of the elastically bonded and bond breaking region (Fig. $2 \mathrm{e}-\mathrm{f}$ ). Note that due to the constant interfacial stress during frictional process, the strain distribution of graphene tends to be triangle-like, allowing us to be characterized based on strain mapping of graphenen nanosheets. This stage ends when the entire interface has entered sliding process, then the interface could not transfer any larger stress into the graphene sheet and the strain of graphene plateaus with the increasing applied strain to the matrix.

\subsection{Mechanical behavior and properties of H-bonded interfaces}

In our experiments, the monolayer graphene nanosheet (with $L \approx 12 \mu \mathrm{m}$ ) was first adhered on top of PMMA matrix via vdW interaction. The strain of graphene sheet was measured along the tensile direction with the aid of Raman spectroscopy when the matrix underwent strain level of $0.1,0.2,0.3,0.4,0.5$ and $0.6 \%$ as shown by the plots in Fig. 3a (see Ref. [39] for more details). It can be seen that the strain builds up in graphene nanosheet from the edges and approaches a peak at the center. At the small applied strain $(<0.3 \%)$, the strain at the center approximately equals the allied strain to the PMMA matrix. And the graphene nanosheet behaved like conventional fibers and its strain distribution could be well described via conventional linear shear-lag analysis or the elastic stage of our model (Fig. 3a black line) [42]. Through eq. (10), we obtain $\beta \approx 0.62 \mu \mathrm{m}^{-1}$, which is commonly treated as an effective parameter presenting the interfacial stress transfer efficiency in fiber/matrix systems [46], and the corresponding interfacial stiffness $k_{e}$ were calculated to be $132 \mathrm{TPa} / \mathrm{m}$, assuming $E_{g}=1 \mathrm{TPa}$. When the applied strain further increases, the strain in the center of graphene begins to lag behind the applied strain to the PMMA matrix. And the strain distribution in graphene becomes almost linear near the edges as labeled by the red line in Fig. 3a, an evidence of the interfacial frictional behavior (interfacial stress tends to be constant at the edges as shown in Fig. $2 \mathrm{e}-\mathrm{f}$ ) as well as nonlinearity for vdW interfaces $[9,11]$. Through fitting the experimental results by the steps in Sec. 4.1, the interfacial strength $\tau_{c}$, the ratio of frictional stress to strength $\alpha$ were found to be $0.45 \mathrm{MPa}$ and 1 , respectively. Our result about the value of interfacial stiffness, strength as well as its relation with the frictional stress $(\alpha=1)$ are similar to that reported previously for a graphene/PET interface
[11]. Particularly, in the terms of frictional stress to strength ratio, the related mechanism was also revealed through molecular dynamics (MD) simulations about vdW interaction dominated graphene interfaces where adhesion and sliding force would spontaneously take place even without an applied normal pressure, resulting in the unique frictional behavior of graphene-based vdW interfaces rather than shear-type interfacial delamination observed in macroscopic interfaces [19]. Meanwhile, its high frictional stress to strength ratio of graphene-based vdW interfaces can also result in huge interfacial energy dissipation during deformation [41,47], as macroscopically achieved high damping performance in nanocarbon based nanocomposites [48-50].

There is increasing evidence in literature for significant improvements in both toughness and strength of graphene-based nanocomposites through engineering their nano-interfaces with hydrogen bonds [26-31], where high interfacial sliding resistance were believed to be one of the most important underlying mechanism. We thus further introduced the $\mathrm{H}$-bonds into the graphene/ PMMA interfacial galleries by following Griegge's mechanism, where the gaseous mixture of ozone and water vapor $\left(\mathrm{H}_{2} \mathrm{O}-\mathrm{O}_{3}\right)$ is jointly involved as oxidizing reagent to oxidize monolayer graphene sheets with hydroxyl, carboxyl and epoxy groups. After $\sim 2$-h reaction, Raman intensity ratio $\left(\mathrm{I}_{\mathrm{D}} / \mathrm{I}_{\mathrm{G}}=\sim 2 ; \mathrm{I}_{\mathrm{D}} / \mathrm{I}_{\mathrm{D}^{\prime}}=\sim 10\right)$ was achieved for the functionalized graphene samples (see Ref. [39] for more details). Following above in situ Raman measurements and nonlinear modelling, it is found that, compared with the pristine $\mathrm{vdW}$ interface, the significant improvements in both the interfacial stiffness $(531 \mathrm{TPa} / \mathrm{m})$ and strength $(1.2 \mathrm{MPa})$ were achieved via hydrogen bonding of the interface as shown in Fig. 3b. This implies that graphene-based interfaces with $\mathrm{H}$-bonding interaction will demonstrate higher interfacial stress transfer efficiency and in turn bulk properties. It is worth noting that the Young's modulus of oxidized graphene was also assumed to be $1 \mathrm{TPa}$, according to the facts that the elastic stiffness of defective graphene $\left(\mathrm{I}_{\mathrm{D}} / \mathrm{I}_{\mathrm{G}}<2\right.$ and $\mathrm{I}_{\mathrm{D}} /$ $\left.\mathrm{I}_{\mathrm{D}^{\prime}}>10\right)$ is not diminished in comparison with its pristine counterpart [51,52]. More interestingly, the overall agreement by fitting with frictional stress of 1.2 MPa ( three times of that for vdW interfaces) for H-bonding graphene/PMMA interfaces is excellent despite some local scattering of the experimental data, strongly supporting their high interfacial sliding forces of $\mathrm{H}$-bonded nanointerfaces. In addition, the fitted frictional stress to strength ratio for H-bonded graphene interface also similar to that observed in previous MD simulation, where the stress-strain curve of $\mathrm{H}$-bonded graphene interfaces displayed little drop after peak stress (frictional stress in close proximity to strength) due to the breaking and re-forming behavior of $\mathrm{H}$-bonds [45]. Moreover, the $\mathrm{H}$-bonded interfaces showed higher sliding resistance in comparison with vdW and even covalent bonded interfaces [45]. It is also worth noting that the damaging stage in our nonlinear model were experimentally undiscernible, which might result from the high value of frictional stress to strength ratio for both vdW and H-bonded interfaces, and on the other hand, also might be experimentally technical limitation e.g. the small size of our measured graphene sheet $(\sim 12 \mu \mathrm{m})$, laser spatial resolution $(1-2 \mu \mathrm{m})$, piezoelectric stage resolution $(\sim 600 \mathrm{~nm})$ as well as the small applied strain $(<1 \%)$. More accurate interfacial parameters thus should be experimentally evaluated via graphene samples with large, size large mismatched deformation and high-resolution characterization for both the vdW and H-bonded interfaces in future.

\subsection{Characteristics of H-bonded interface in graphene-based nanocomposites}

There are important implications from this study for optimal design of graphene-based high-performance nanocomposites 

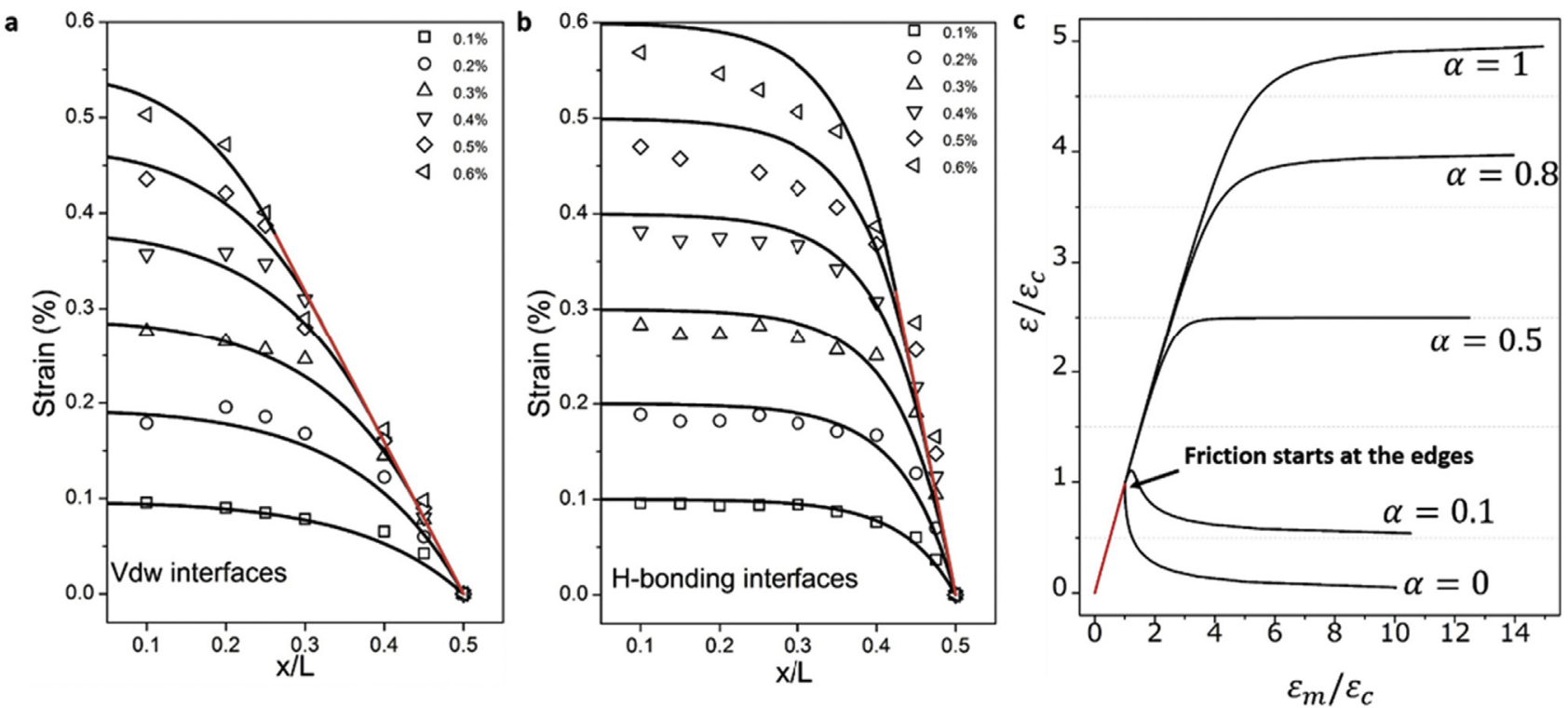

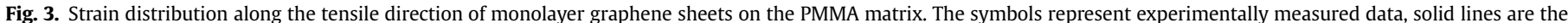

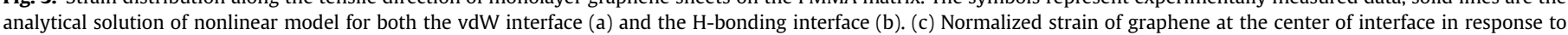

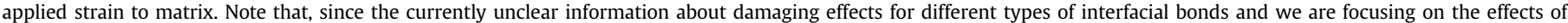
frictional behavior on the reinforcing mechanism, the $k_{d}=\infty$ is assumed for simplicity in the present analysis.

through engineering their nano-interfaces with hydrogen bonds. On the basis of our results as well as previous reports, we conclude here the beneficial characteristics of $\mathrm{H}$-bonded interface in improve the overall properties of graphene-based or nano-carbon-based nanocomposites. i) After introduction of $\mathrm{H}$-bond-required oxide groups on graphene nanosheet, defective graphene is still capable of tolerating defects and retaining its ultra-high stiffness properties when $\mathrm{I}_{\mathrm{D}} / \mathrm{I}_{\mathrm{G}}<4$ (an $\mathrm{I}_{\mathrm{D}} / \mathrm{I}_{\mathrm{G}}$ ratio of 1 is generally considered to represent a relatively high state of defectiveness in graphene) [51]. ii) As proven in section 4.2, compared with pristine interface, significant improvements in both the interfacial stiffness and strength could be achieved via hydrogen bonding, which are critical for the bulk modulus and strength of nanocomposites. iii) H-bondrequired oxide groups further featured interfacial galleries with richer binding chemistry, allowing further $\mathrm{H}$-bonding network tuning as well as combination with other type of bonds (i.e. strong covalent bonds and coordinative bonds) and in turn, leading to improved interfacial properties [22,53,54]. iv) Owing to their short, unidirectional characteristics, the H-bonds between the graphene nanosheets and polymer matrix could be able to re-form after breaking under deformation, resulting in a high frictional stress (comparable to the interfacial strength). Comparatively, directional and strong covalent bonds take the advantages in terms of ii) whereas they are hard to reform after breaking, limiting the reinforcing effects of graphene or nano-carbon materials in nanocomposites [44]. Such effects of frictional behavior on the overall properties of nanocomposites could also be easily speculated from the strain of graphene at the center of interface in response to applied strain to matrix as shown in Fig. 3c. Once the applied strain reaches the critical value, interfacial slippage initiates at the edge and propagates to the center of graphene as above-analyzed. It can be found that, for interfaces (e.g. covalent bonded interfaces) with a
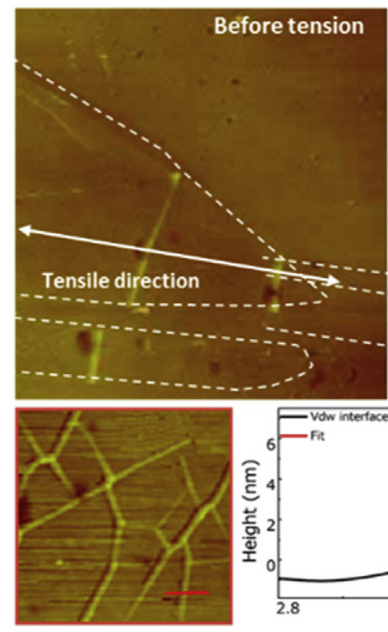

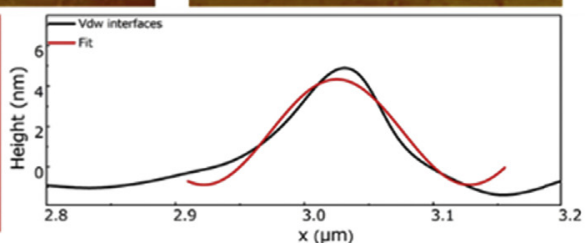

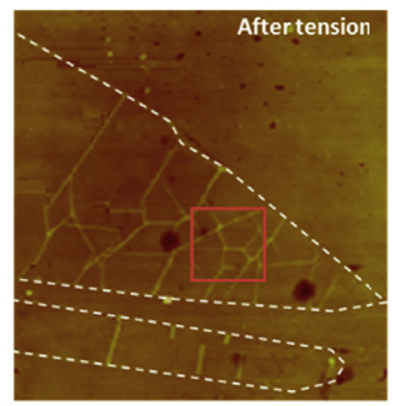
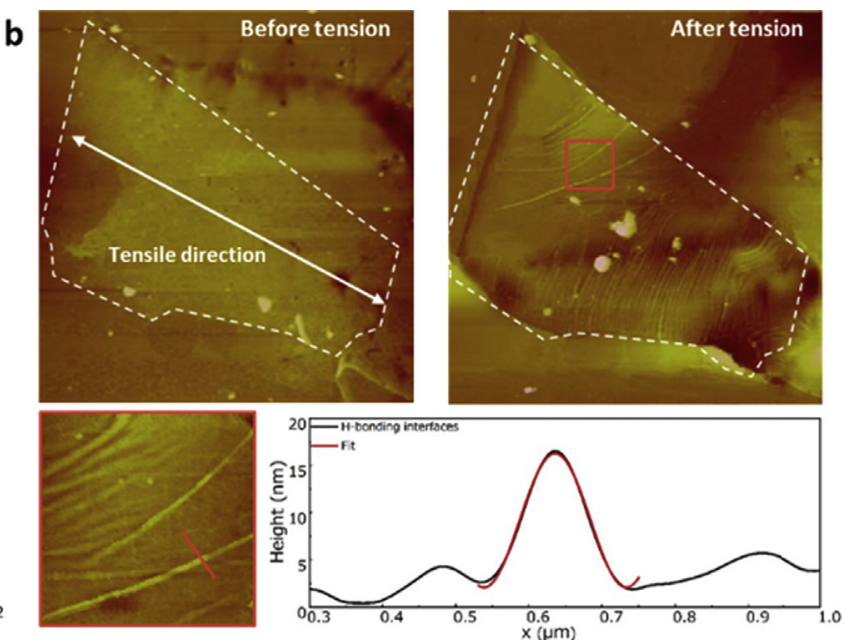

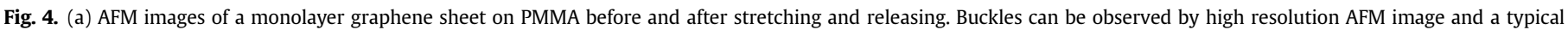
profile across the buckles on the graphene sheets is plotted. (b) The strain at the middle of graphene sheet with respect to the applied strain onto the matrix. 
low frictional stress-strength ratio [45], the strain of graphene at the center will decrease, instead of increase with applied strain, hence weakening their strengthening effects. Nevertheless, for interfaces with $\mathrm{H}$-bonded interaction (or high frictional stressstrength ratio), the graphene still keeps their strengthening effect without losing initial efficiency for a large strain level after the bonds break at the edge as shown in Fig. 3c [55]. Meanwhile, during their repeatedly breaking and re-forming processes of interfacial bonds, considerable mechanical energy is dissipated, which could further attribute to the bulk toughness and damping properties of nanocomposites [25-30].

\subsection{Interfacial adhesion energy of H-bonded graphene/polymer nano-Interfaces}

The monolayer graphene sheet was exposed on top of PMMA matrix as illustrated in Fig. 1a, also allowing the morphology characterization of graphene sheet via before and after tensile deformation of matrix. Since frictional behaver of graphene-based interfaces occurs, matrix will create the compressive restraints on the graphene nanosheets after releasing the applied strain, leading to wrinkling or buckle delamination in order to release the compressive strain [11]. Herein, atomic force microscope (AFM) was employed to detect the surface in our graphene samples after loading-unloading process. Buckling ridges, localized with relatively flat surface between neighboring ridges (characteristics of buckle delamination), were observed in both vdW and H-bonded interface in Fig. 4 [11,56,57]. We hence calculate the adhesion energy of graphene-based interfaces (also known as the interface toughness) based on fracture mechanics, which offers a simple and strain-independent formula as [56]:

$\Gamma=\frac{\pi^{4}}{32} \frac{E_{g} t \delta^{4}}{\lambda^{4}}+\frac{\pi^{4}}{6} \frac{t^{3} \delta^{2}}{\lambda^{4}} \frac{E_{g}}{1-\nu^{2}}$

where $T$ is the adhesion energy, $\delta$ is the delamination height, and $\lambda$ is the delamination width, $\nu$ is Poisson's ratio of the graphene nanosheet. Based on more than six buckles observed in Fig. 4 a and b, we estimated $\delta=8.1 \mathrm{~nm}, \lambda=199 \mathrm{~nm}$ for graphene-based interface under vdW interaction and $\delta=17.2 \mathrm{~nm}, \lambda=181 \mathrm{~nm}$ for that under H-bonding interaction via a sine-type fitting [57]. After assuming $E_{g}=1 \mathrm{TPa}, \nu=0.16$ and $t=0.34 \mathrm{~nm}$ for graphene nanosheets [3], the adhesion energy of vdW and H-bonded graphene/PMMA interfaces could be estimated to be 2.8 and $84.4 \mathrm{~mJ} /$ $\mathrm{m}^{-2}$, respectively, further highlighting the significantly improvements in the interfacial properties through $\mathrm{H}$-bonding treatment.

\section{Conclusion}

Based on the in situ Raman measurements and nonlinear shearlag model, we study the stress transfer of hydrogen bonded graphene/PMMA nano-interface. A nonlinear shear-lag model and corresponding analytical solutions were presented to understand evolution of interfacial (shear) stresses along the interface. Our nonlinear model might be also valid for graphene nano-interfaces with various types of interfacial interactions. The quantified interfacial parameters (stiffness, strength, frictional stress and toughness) for H-bonded interface can be substituted into micromechanical models to give the relationship between more complicated structures and overall properties of bulk composites. Implications for optimal design of graphene-based high-performance nanocomposites through tuning their nano-interfaces with hydrogen bonds were further summarized. Also, the understanding of the mechanical behavior and properties of $\mathrm{H}$-bonded graphene/ polymer interface here could be useful in developing a basis for interfacial optimal design of high-performance graphene-based nanocomposites.

\section{Acknowledgement}

This project was jointly supported by the National Key Basic Research Program of China (Grant Nos 2012 CB937503 and 2013Св934203) and the National Natural Science Foundation of China (Grant Nos. 51173030, 11225210, 21474023, 11222217, 11225421,11434010 and 11474277).

\section{References}

[1] A.K. Geim, K.S. Novoselov, The rise of graphene, Nat. Mater. 6 (2007) 183-191.

[2] S. Iijima, T. Ichihashi, Single-shell carbon nanotubes of 1-nm diameter, Nature 363 (1993) 603-605.

[3] C. Lee, X. Wei, J.W. Kysar, J. Hone, Measurement of the elastic properties and intrinsic strength of monolayer graphene, Science 321 (2008) 385-388.

[4] M.-F. Yu, O. Lourie, M.J. Dyer, K. Moloni, T.F. Kelly, R.S. Ruoff, Strength and breaking mechanism of multiwalled carbon nanotubes under tensile load, Science 287 (2000) 637-640.

[5] S. Stankovich, D.A. Dikin, G.H. Dommett, K.M. Kohlhaas, E.J. Zimney, E.A. Stach, R.D. Piner, S.T. Nguyen, R.S. Ruoff, Graphene-based composite materials, Nature 442 (2006) 282-286.

[6] A.H. Barber, S.R. Cohen, S. Kenig, H.D. Wagner, Interfacial fracture energy measurements for multi-walled carbon nanotubes pulled from a polymer matrix, Compos. Sci. Technol. 64 (15) (2004) 2283-2289.

[7] H.D. Wagner, R.A. Vaia, Nanocomposites: issues at the interface, Mater. Today 7 (2004) 38-42.

[8] A.H. Barber, S.R. Cohen, A. Eitan, L.S. Schadler, H.D. Wagner, Fracture transitions at a carbon-nanotube/polymer interface, Adv. Mater. 18 (2006) 83-87.

[9] L. Gong, I.A. Kinloch, R.J. Young, I. Riaz, R. Jalil, K.S. Novoselov, Interfacial stress transfer in a graphene monolayer nanocomposite, Adv. Mater. 22 (2010) 2694-2697.

[10] R.J. Young, I.A. Kinloch, L. Gong, K.S. Novoselov, The mechanics of graphene nanocomposites: a review, Compos. Sci. Technol. 72 (12) (2012) 1459-1476.

[11] T. Jiang, R. Huang, Y. Zhu, Interfacial sliding and buckling of monolayer graphene on a stretchable substrate, Adv. Funct. Mater. 24 (2014) 396-402.

[12] A.P.A. Raju, A. Lewis, B. Derby, R.J. Young, I.A. Kinloch, R. Zan, K.S. Novoselov, Wide-area strain sensors based upon graphene-polymer composite coatings probed by raman spectroscopy, Adv. Funct. Mater. 24 (2014) 2865-2874.

[13] G. Wang, L. Liu, Z. Dai, Q. Liu, H. Miao, Z. Zhang, Biaxial compressive behavior of embedded monolayer graphene inside flexible poly (methyl methacrylate) matrix, Carbon 86 (2015) 69-77.

[14] I. Srivastava, R.J. Mehta, Z.-Z. Yu, L. Schadler, N. Koratkar, Raman study of interfacial load transfer in graphene nanocomposites, Appl. Phys. Lett. 98 (2011) 063102.

[15] Z. Li, I.A. Kinloch, R.J. Young, K.S. Novoselov, G. Anagnostopoulos, J. Parthenios, C. Galiotis, K. Papagelis, C.-Y. Lu, L. Britnell, The deformation of wrinkled graphene, ACS Nano 9 (2015) 3917-3925.

[16] G. Tsoukleri, J. Parthenios, K. Papagelis, R. Jalil, A.C. Ferrari, A.K. Geim, K.S. Novoselov, C. Galiotis, Subjecting a graphene monolayer to tension and compression, Small 5 (2009) 2397-2402.

[17] C. Galiotis, Interfacial studies on model composites by laser Raman spectroscopy, Compos. Sci. Technol. 42 (1991) 125-150.

[18] G. Guo, Y. Zhu, Cohesive-shear-lag modeling of interfacial stress transfer between a monolayer graphene and a polymer substrate, J. Appl. Mech. 82 (2015) 031005.

[19] N.M. Pugno, Q. Yin, X. Shi, R. Capozza, A generalization of the Coulomb's friction law: from graphene to macroscale, Meccanica 48 (2013) 1845-1851.

[20] Q. Cheng, J. Duan, Q. Zhang, L. Jiang, Learning from nature: constructing integrated graphene-based artificial nacre, ACS Nano 9 (2015) 2231-2234.

[21] B.L. Smith, T.E. Schäffer, M. Viani, J.B. Thompson, N.A. Frederick, J. Kindt A. Belcher, G.D. Stucky, D.E. Morse, P.K. Hansma, Molecular mechanistic origin of the toughness of natural adhesives, fibres and composites, Nature 399 (1999) 761-763.

[22] N.V. Medhekar, A. Ramasubramaniam, R.S. Ruoff, V.B. Shenoy, Hydrogen bond networks in graphene oxide composite paper: structure and mechanical properties, ACS Nano 4 (2010) 2300-2306.

[23] O.C. Compton, S.W. Cranford, K.W. Putz, Z. An, L.C. Brinson, M.J. Buehler S.T. Nguyen, Tuning the mechanical properties of graphene oxide paper and its associated polymer nanocomposites by controlling cooperative intersheet hydrogen bonding, ACS nano 6 (2012) 2008-2019.

[24] Y.L. Liu, B. Xie, Z. Zhang, Q.S. Zheng, Z.P. Xu, Mechanical properties of graphene papers, J. Mech. Phys. Solids 60 (2012) 591-605.

[25] Y. Gao, L.Q. Liu, S.Z. Zu, K. Peng, D. Zhou, B.H. Han, Z. Zhang, The effect of interlayer adhesion on the mechanical behaviors of macroscopic graphene oxide papers, ACS Nano 5 (2011) 2134-2141.

[26] H. Zhu, S. Zhu, Z. Jia, S. Parvinian, Y. Li, O. Vaaland, L. Hu, T. Li, Anomalous 
scaling law of strength and toughness of cellulose nanopaper, Proc. Natl. Acad. Sci. 112 (29) (2015) 8971-8976.

[27] R. Rafiq, D. Cai, J. Jin, M. Song, Increasing the toughness of nylon 12 by the incorporation of functionalized graphene, Carbon 48 (2010) 4309-4314.

[28] K.W. Putz, O.C. Compton, M.J. Palmeri, S.T. Nguyen, L.C. Brinson, High-nanofiller-content graphene oxide-polymer nanocomposites via vacuum-assisted self-assembly, Adv. Funct. Mater. 20 (2010) 3322-3329.

[29] Z. Xu, H. Sun, X. Zhao, C. Gao, Ultrastrong fibers assembled from giant graphene oxide sheets, Adv. Mater. 25 (2013) 188-193.

[30] J. Liu, C. Chen, C. He, J. Zhao, X. Yang, H. Wang, Synthesis of graphene peroxide and its application in fabricating super extensible and highly resilient nanocomposite hydrogels, ACS nano 6 (2012) 8194-8202.

[31] W. Ma, L. Liu, R. Yang, T. Zhang, Z. Zhang, L. Song, Y. Ren, J. Shen, Z. Niu, W. Zhou, Monitoring a micromechanical process in macroscale carbon nanotube films and fibers, Adv. Mater. 21 (2009) 603-608.

[32] W. Ma, L. Liu, Z. Zhang, R. Yang, G. Liu, T. Zhang, X. An, X. Yi, Y. Ren, Z. Niu, J. Li, H. Dong, W. Zhou, P.M. Ajayan, S. Xie, High-strength composite fibers: real izing true potential of carbon nanotubes in polymer matrix through continuous reticulate architecture and molecular level couplings, Nano Lett. 9 (2009) 2855-2861.

[33] Y. Gao, J. Li, L. Liu, W. Ma, W. Zhou, S. Xie, Z. Zhang, Axial compression of hierarchically structured carbon nanotube fiber embedded in epoxy, Adv. Funct. Mater. 20 (2010) 3797-3803.

[34] J. Li, Y. Gao, W. Ma, L. Liu, Z. Zhang, Z. Niu, Y. Ren, X. Zhang, Q. Zeng, H. Dong, High performance, freestanding and superthin carbon nanotube/epoxy nanocomposite films, Nanoscale 3 (2011) 3731-3736.

[35] L. Liu, Y. Gao, Q. Liu, J. Kuang, D. Zhou, S. Ju, B. Han, Z. Zhang, High mechanica performance of layered graphene oxide/poly (vinyl alcohol) nanocomposite films, Small 9 (2013) 2466-2472.

[36] Q. Liu, L. Liu, K. Xie, Y. Meng, H. Wu, G. Wang, Z. Dai, Z. Wei, Z. Zhang, Synergistic effect of r-GO/PANI nanocomposite electrode based air working ionic actuator with a large actuation stroke and long-term durability, J. Mater. Chem. A 3 (2015) 8380-8388.

[37] J.Shang, Y.Chen, Y.Zhou, L. Liu, G. Wang, X. Li, J. Kuang, Q. Liu, Z. Dai, H. Miao, Effect of folded and crumpled morphologies of graphene oxide platelets on the mechanical performances of polymer nanocomposites, Polymer 68 (2015) 131-139.

[38] K. Peng, L.-Q. Liu, H. Li, H. Meyer, Z. Zhang, Room temperature functionalization of carbon nanotubes using an ozone/water vapor mixture, Carbon 49 (2011) 70-76.

[39] G. Wang, Z. Dai, L. Liu, H. Hu, Q. Dai, Z. Zhang, Tuning interfacial adhesion and its impact on reinforcing mechanisms for monolayer graphene/polymer nanocomposites, ACS Appl. Mater. Interf. 8 (2016) 22554-22562.

[40] A.H. Barber, S.R. Cohen, H.D. Wagner, Measurement of carbon nanotube-polymer interfacial strength, Appl. Phys. Lett. 82 (2003) 4140-4142.
[41] B. Ji, H. Gao, Mechanical properties of nanostructure of biological materials, J. Mech. Phys. Solids 52 (2004) 1963-1990.

[42] H. Cox, The elasticity and strength of paper and other fibrous materials, Br. J. Appl. Phys. 3 (1952) 72.

[43] L. De Lorenzis, G. Zavarise, Cohesive zone modeling of interfacial stresses in plated beams, Int. J. Solids Struct. 46 (2009) 4181-4191.

[44] Y. Liu, B. Xie, Z. Xu, Mechanics of coordinative crosslinks in graphene nanocomposites: a first-principles study, J. Mater. Chem. 21 (2011) 6707-6712.

[45] Y.L. Liu, Z.P. Xu, Multimodal and self-healable interfaces enable strong and tough graphene-derived materials, J. Mech. Phys. Solids 70 (2014) 30-41.

[46] C. Galiotis, A. Paipetis, Definition and measurement of the shear-lag parameter, $\beta$, as an index of the stress transfer efficiency in polymer composites, J. Mater. Sci. 33 (1998) 1137-1143.

[47] Y. Ni, Z. Song, H. Jiang, S.-H. Yu, L. He, Optimization design of strong and tough nacreous nanocomposites through tuning characteristic lengths, J. Mech. Phys. Solids 81 (2015) 41-57.

[48] N. Koratkar, B.Q. Wei, P.M. Ajayan, Carbon nanotube films for damping applications, Adv. Mater. 14 (2002) 997-1000.

[49] J. Suhr, W. Zhang, P.M. Ajayan, N.A. Koratkar, Temperature-activated interfacial friction damping in carbon nanotube polymer composites, Nano Lett. 6 (2006) 219-223.

[50] J. Suhr, N. Koratkar, P. Keblinski, P. Ajayan, Viscoelasticity in carbon nanotube composites, Nat. Mater. 4 (2005) 134-137.

[51] A. Zandiatashbar, G.-H. Lee, S.J. An, S. Lee, N. Mathew, M. Terrones, T. Hayashi, C.R. Picu, J. Hone, N. Koratkar, Effect of defects on the intrinsic strength and stiffness of graphene, Nat. Commun. 5 (2014) 3186.

[52] G. López-Polín, C. Gómez-Navarro, V. Parente, F. Guinea, M.I. Katsnelson, F. Pérez-Murano, J. Gómez-Herrero, Increasing the elastic modulus of graphene by controlled defect creation, Nat. Phys. 11 (2015) 26-31.

[53] D.A. Dikin, S. Stankovich, E.J. Zimney, R.D. Piner, G.H. Dommett, G. Evmenenko, S.T. Nguyen, R.S. Ruoff, Preparation and characterization of graphene oxide paper, Nature 448 (2007) 457-460.

[54] S. Park, K.-S. Lee, G. Bozoklu, W. Cai, S.T. Nguyen, R.S. Ruoff, Graphene oxide papers modified by divalent ions-Enhancing mechanical properties via chemical cross-linking, ACS Nano 2 (2008) 572-578.

[55] Z. Shuchun, W. Yueguang, Effective elastic modulus of bone-like hierarchical materials, Act. Mech. Sol. Sin. 20 (2007) 198-205.

[56] S. Faulhaber, C. Mercer, M.-W. Moon, J. Hutchinson, A. Evans, Buckling delamination in compressed multilayers on curved substrates with accompanying ridge cracks, J. Mech. Phys. Solids 54 (2006) 1004-1028.

[57] C.J. Brennan, J. Nguyen, E.T. Yu, N. Lu, Interface adhesion between 2D materials and elastomers measured by buckle delaminations, Adv. Mater. Interf. 2 (2015) 1500176. 